\title{
Grading of hirsutism: a practical approach to the modified Ferriman-Gallwey scoring system
}

\author{
Filiz Cebeci Kahraman¹, Sevil Savaş Erdoğan²
}

${ }^{1}$ Department of Dermatology, Istanbul Medeniyet University, Göztepe Prof. Dr. Süleyman Yalçın City Hospital, Istanbul, Turkey 2Department of Dermatology, University of Health Sciences, Sultan 2. Abdulhamid Han Training and Research Hospital, Istanbul, Turkey

Adv Dermatol Allergol 2022; XXXIX (4): 744-748

DOI: https://doi.org/10.5114/ada.2021.108455

\begin{abstract}
Introduction: The modified Ferriman-Gallwey (mFG) scale is accepted as a useful clinical scoring system in the evaluation of hirsutism.

Aim: To prevent overlooking hirsutism diagnoses and facilitate patient examinations in ongoing pandemic conditions by simplifying the mFG score.

Material and methods: This study included 227 patients who were diagnosed with hirsutism and had mFG scores of $\geq 8$. Hair distribution and hair growth intensity of nine different body regions in the $\mathrm{mFG}$ score were examined. Results: Among our patients with hirsutism, terminal hair was present on the chin in $97.4 \%(n=221)$, thighs in $96.5 \%(n=219)$, upper lip in $94.7 \%(n=215)$, and lower abdomen in $92.1 \%(n=205)$, and this was significantly higher compared with the remaining five regions ( $p<0.001$ for each comparison). Terminal hair growth scored as $\geq 1$ was found in $89 \%$ of the patients $(n=202)$ for the combination of the chin, thighs, and upper lip, and in $87.2 \%$ $(n=198)$ for the combination of the chin, thighs, and lower abdomen. When the chin and thighs were evaluated together, $75.3 \%(n=171)$ of the patients had $\geq 2$ terminal hair growth.

Conclusions: In addition to the chin and thighs being the main regions of terminal hair growth, examination of the upper lip or lower abdomen can sufficiently help predict hirsutism. During the ongoing pandemic conditions when we need to follow social distancing rules, a practical approach to the mFG scoring system will facilitate the work of many physicians, including dermatologists, and will shorten the patient's stay in the clinic.
\end{abstract}

Key words: Ferriman-Gallwey score, hair growth, hirsutism.

\section{Introduction}

Hirsutism is defined as the overgrowth of male-type terminal hair in the androgen-sensitive areas of women [1]. Approximately $5-10 \%$ of women of reproductive age have hirsutism [2]. More than $80 \%$ of women with hirsutism have polycystic ovary syndrome (PCOS), and approximately 10\% have idiopathic hirsutism [3]. Therefore, the body examination of patients presenting with excessive hair growth, acne, androgenetic alopecia, and menstrual irregularities should not be neglected to detect hirsutism.

The methods used for the clinical evaluation of hirsutism include photographic evaluation, microscopic measurements of hair diameters, and computer-based measurement of photographed hair [4]. However, grading of hair growth according to the modified FerrimanGallwey ( $\mathrm{mFG}$ ) scale is a simpler and widely used stan- dard method based on a visual scoring system [1]. The original system was based on the scoring of the presence of hair in 11 regions, which was then modified to include nine regions [5].

Although the mFG scale is simpler than other methods, it involves the evaluation of the amount of terminal hair in nine androgen-sensitive body regions: the upper lip, chin, chest, upper and lower back, upper and lower abdomen, upper arm, and thighs. The ongoing pandemic, which is present all over the world, has brought about certain difficulties in patient examinations, especially concerning methods that require full undressing. The evaluation of all these nine regions is challenging for both physicians and patients in dermatology outpatient clinics where time is limited and patient density is high. The purpose of this study was to shorten the duration of contact with the patient during the examination and

Address for correspondence: Filiz Cebeci Kahraman Assoc. Prof., Department of Dermatology, Istanbul Medeniyet University, Göztepe Prof. Dr. Süleyman Yalçın City Hospital, Dr. Erkin Street, Kadıköy, 347134 Istanbul, Turkey, phone: +90 5373724722 , fax: +90 2122340419 , e-mail: cebecifiliz@yahoo.com

Received: 25.05.2021, accepted: 22.06.2021. 
to simplify examinations for the detection of hirsutism in socioculturally conservative countries, such as Turkey. The examination of these nine regions, which present with higher hair density, can provide rapid assessment, reduce contact with the patient, and prompt physicians to examine other regions if necessary.

\section{Aim}

Therefore, we aimed to identify a more practical method that could be used in screening women suspected of having hirsutism by revealing the body regions where hair growth was clustered.

\section{Material and methods}

The study group consisted of 227 women aged 18-45 years who presented to our dermatology outpatient clinic and were diagnosed with hirsutism. The study was conducted according to the principles of the Declaration of Helsinki and approved by the local ethics committee. Women with an mFG score of $\geq 8$ and diagnosis of idiopathic hirsutism or PCOS were included in the study, retrospectively. The diagnosis of idiopathic hirsutism was made according to the presence of hirsutism, regular menstrual cycle, and normal serum androgen profile [6]. The diagnosis of PCOS was made according to the revised Rotterdam diagnostic criteria based on the presence of at least two of the following three criteria: oligo- or anovulation; clinical and/or biochemical signs of hyperandrogenism; and polycystic ovaries and exclusion of other related disorders [7]. Pregnant or lactating patients, those who used drugs such as oral contraceptives, corticosteroids, cyclosporine or spironolactone within the last 3 months, and those with chronic diseases, thyroid hormone dysfunction, congenital adrenal hyperplasia, adrenal or ovarian tumours and other endocrine disorders were excluded from the study.

According to the mFG scoring system, each region was separately evaluated in terms of the rate of terminal hair growth and scored from 0 (absence) to 4 (excessive). The total score was calculated, and an $\mathrm{mFG}$ score of $\geq 8$ was accepted as hirsutism [1, 5]. The severity of hirsutism was classified as mild if the MFG score was 8-16, moderate if 17-24, and severe if above 24 [1]. The details of the mFG scores and age were retrospectively obtained from the files of the patients. The presence and intensity of hair growth in all regions were determined separately by a single physician.

\section{Statistical analysis}

The SPSS v. 15.0 for Windows software package was used for statistical analyses. Descriptive statistics are given as numbers and percentages for categorical variables, and mean, standard deviation, minimum, maximum, median and interquartile range for numerical variables.
Scores were compared using the Friedman test in more than two dependent groups. Subgroup analyses were performed using the Wilcoxon test. The comparison of rates between more than two dependent groups was undertaken using Cochran's Q test. Subgroup analysis was performed using the McNemar's test. The results were interpreted using Bonferroni correction in subgroup analyses of more than two groups. The rates between the independent groups were compared using the $\chi^{2}$ test. The comparisons of numerical variables in independent groups were performed using the Mann-Whitney $U$ test because the conditions of normal distribution were not met. The statistical significance level of $\alpha$ was accepted as $p<0.05$.

\section{Results}

In this study, 227 patients with hirsutism were evaluated. The mean age of the women with hirsutism was $25.4 \pm 6$ (range: $18-45$ ) years. The mean total $m F G$ score was $12.9 \pm 4.2$ (range: $8-35)$, and $80.6 \%(n=183)$ of the patients had mild, $18.5 \%(n=42)$ moderate, $0.9 \%(n=2)$ severe hirsutism. The average hair growth scores by regions were $2.85 \pm 1.08$ for the chin, $2.86 \pm 1.15$ for the thighs, $1.99 \pm 1.10$ for the lip, $2.08 \pm 1.04$ for the lower abdomen, $1.48 \pm 0.99$ for the chest, $0.77 \pm 1.08$ for the lower back, $0.35 \pm 0.70$ for the upper abdomen, $0.35 \pm 0.68$ for the arm, and $0.19 \pm 0.62$ for the upper back (Table 1).

Table 1 shows the percentages of hair growth scores for the nine regions included in the $\mathrm{mFG}$ scoring system among the women in our study group (Figure 1). Terminal hair was present on the chin in $97.4 \%(n=221)$ of the patients, thighs in 96.5\% ( $n=219)$, upper lip in $94.7 \%$ $(n=215)$, and lower abdomen in 92.1\% $(n=205)$ (Figure 2). The rates of the presence of terminal hair on the chin, upper lip, lower abdomen, and thighs (i.e., grade 1) were statistically significantly higher when compared with the remaining regions (chest, upper abdomen, lower back, upper back, and upper arm) ( $p<0.001$ for each comparison; $p<0.001$ for the subgroup analysis with the Bonferroni correction).

Table 2 shows the distribution of patients with hair growth scores of $\geq 2, \geq 3$, and 4 by region. Accordingly, the hair growth score was 2 or above for the chin region in $88.1 \%$ of the patients $(n=200)$, thighs in $85.9 \%(n=195)$, lower abdomen in $72.2 \%(n=164)$, upper lip in $62.1 \%(n=$ $141)$, and chest in $48 \%(n=109)$ (Figure 3$)$. The presence of terminal hair scored as $\geq 2$ on the chin, upper lip, chest, lower abdomen, and thighs were statistically significantly higher when compared with the remaining regions (upper abdomen, lower back, upper back, and upper arm) ( $p<$ 0.001 for each comparison and $p<0.001$ for the subgroup analysis with Bonferroni correction) (Table 2).

When we evaluated the regions according to the highest presence of hair growth, the rate of patients with terminal hair scored as $\geq 1$ was $89 \%(n=202)$ for 
Table 1. Hair growth scores by body region

\begin{tabular}{|c|c|c|c|c|c|c|c|c|}
\hline \multirow[t]{2}{*}{ Body region } & \multicolumn{8}{|c|}{ Hair growth score } \\
\hline & Mean \pm SD & Median (IQR) & & 0 & 1 & 2 & 3 & 4 \\
\hline \multirow[t]{2}{*}{ Chin } & $2.85 \pm 1.08$ & $3(2-4)$ & $n$ & 6 & 21 & 52 & 70 & 78 \\
\hline & & & $\%$ & 2.6 & 9.3 & 22.9 & 30.8 & 34.4 \\
\hline \multirow[t]{2}{*}{ Thigh } & $2.86 \pm 1.15$ & $3(2-4)$ & $n$ & 8 & 24 & 47 & 60 & 88 \\
\hline & & & $\%$ & 3.5 & 10.6 & 20.7 & 26.4 & 38.8 \\
\hline \multirow[t]{2}{*}{ Upper lip } & $1.99 \pm 1.10$ & $2(1-3)$ & $\mathrm{n}$ & 12 & 74 & 74 & 39 & 28 \\
\hline & & & $\%$ & 5.3 & 32.6 & 32.6 & 17.2 & 12.3 \\
\hline \multirow[t]{2}{*}{ Lower abdomen } & $2.08 \pm 1.04$ & $2(1-3)$ & $\mathrm{n}$ & 18 & 45 & 80 & 69 & 15 \\
\hline & & & $\%$ & 7.9 & 19.8 & 35.2 & 30.4 & 6.6 \\
\hline \multirow[t]{2}{*}{ Chest } & $1.48 \pm 0.99$ & $1(1-2)$ & $\mathrm{n}$ & 39 & 79 & 74 & 31 & 4 \\
\hline & & & $\%$ & 17.2 & 34.8 & 32.6 & 13.7 & 1.8 \\
\hline \multirow[t]{2}{*}{ Lower back } & $0.77 \pm 1.08$ & $0(0-1)$ & $\mathrm{n}$ & 126 & 59 & 18 & 17 & 7 \\
\hline & & & $\%$ & 55.5 & 26.0 & 7.9 & 7.5 & 3.1 \\
\hline \multirow[t]{2}{*}{ Upper abdomen } & $0.35 \pm 0.70$ & $0(0-1)$ & $\mathrm{n}$ & 169 & 42 & 11 & 4 & 1 \\
\hline & & & $\%$ & 74.4 & 18.5 & 4.8 & 1.8 & 0.4 \\
\hline \multirow[t]{2}{*}{ Arm } & $0.35 \pm 0.68$ & $0(0-1)$ & $n$ & 169 & 42 & 12 & 3 & 1 \\
\hline & & & $\%$ & 74.4 & 18.5 & 5.3 & 1.3 & 0.4 \\
\hline \multirow[t]{2}{*}{ Upper back } & $0.19 \pm 0.62$ & $0(0-0)$ & $\mathrm{n}$ & 201 & 16 & 5 & 3 & 2 \\
\hline & & & $\%$ & 88.5 & 7.0 & 2.2 & 1.3 & 0.9 \\
\hline
\end{tabular}

$S D$ - standard deviation, IQR - interquartile range. Subgroup analysis with Bonferroni correction, $p<0.001$.

the combination of the chin, thigh, and lip regions and $87.2 \%(n=198)$ for the combination of chin, thigh, and lower abdomen regions. The most common regions with $\geq 2$ terminal hair growth scores in our patients were the chin and thighs. The proportion of patients with terminal hair growth score $\geq 2$ on both chin and thighs $75.3 \%(n=171)$.

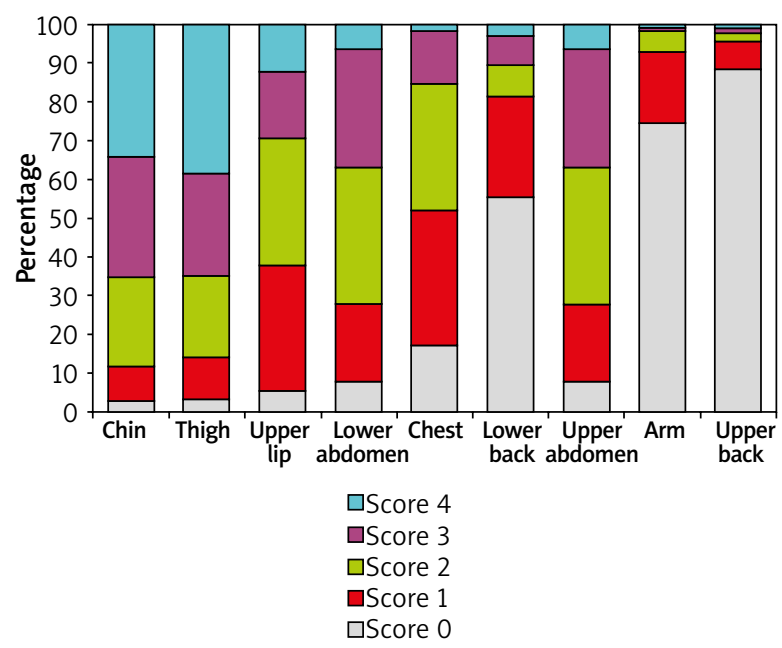

Figure 1. Distribution of hair growth scores according to the body region

\section{Discussion}

In this study, we examined the distribution and density of terminal hair in nine body regions, and the results showed that the chin, thighs, upper lip, and lower abdomen were the regions that most contributed to the total body hair growth score in the Turkish population. Given that our study group comprised only patients with hirsutism and we observed less frequency and intensity of hair growth in the upper abdomen, lower back, upper back and upper arm in these patients, we can easily state that these four regions can be disregarded when using the mFG scoring system in Turkish patients.

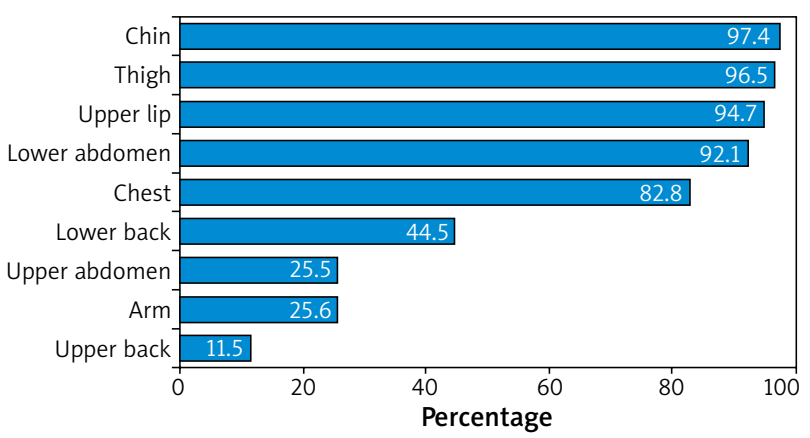

Figure 2. Distribution of patients with a hair growth score of $\geq 1$ according to the body regions 
Table 2. Distribution of patients according to hair growth scores by body region

\begin{tabular}{|c|c|c|c|c|c|c|c|c|c|}
\hline \multirow[t]{2}{*}{ Body region } & & \multicolumn{2}{|c|}{ Score $\geq 1$} & \multicolumn{2}{|c|}{ Score $\geq 2$} & \multicolumn{2}{|c|}{ Score $\geq 3$} & \multicolumn{2}{|c|}{ Score $=4$} \\
\hline & & Absent & Present & Absent & Present & Absent & Present & Absent & Present \\
\hline \multirow[t]{2}{*}{ Chin } & $n$ & 6 & 221 & 27 & 200 & 79 & 148 & 149 & 78 \\
\hline & $\%$ & 2.6 & 97.4 & 11.9 & 88.1 & 34.8 & 65.2 & 65.6 & 34.4 \\
\hline \multirow[t]{2}{*}{ Thigh } & $n$ & 8 & 219 & 32 & 195 & 79 & 148 & 139 & 88 \\
\hline & $\%$ & 3.5 & 96.5 & 14.1 & 85.9 & 34.8 & 65.2 & 61.2 & 38.8 \\
\hline \multirow[t]{2}{*}{ Upper lip } & $n$ & 12 & 215 & 86 & 141 & 160 & 67 & 199 & 28 \\
\hline & $\%$ & 5.3 & 94.7 & 37.9 & 62.1 & 70.5 & 29.5 & 87.8 & 12.3 \\
\hline \multirow[t]{2}{*}{ Lower abdomen } & $n$ & 18 & 209 & 63 & 164 & 143 & 84 & 212 & 15 \\
\hline & $\%$ & 7.9 & 92.1 & 27.8 & 72.2 & 63.0 & 37.0 & 93.4 & 6.6 \\
\hline \multirow[t]{2}{*}{ Chest } & $n$ & 39 & 188 & 118 & 109 & 192 & 35 & 223 & 4 \\
\hline & $\%$ & 17.2 & 82.8 & 52.0 & 48.0 & 84.6 & 15.4 & 98.2 & 1.8 \\
\hline \multirow[t]{2}{*}{ Lower back } & $n$ & 126 & 101 & 185 & 42 & 203 & 24 & 220 & 7 \\
\hline & $\%$ & 55.5 & 44.5 & 81.5 & 18.5 & 89.4 & 10.6 & 96.9 & 3.1 \\
\hline \multirow[t]{2}{*}{ Upper abdomen } & $n$ & 169 & 58 & 211 & 16 & 222 & 5 & 226 & 1 \\
\hline & $\%$ & 74.4 & 25.6 & 93.0 & 7.0 & 97.8 & 2.2 & 99.6 & 0.4 \\
\hline \multirow[t]{2}{*}{ Arm } & $n$ & 169 & 58 & 211 & 16 & 223 & 4 & 226 & 1 \\
\hline & $\%$ & 74.4 & 25.6 & 93.0 & 7.0 & 98.2 & 1.8 & 99.6 & 0.4 \\
\hline \multirow[t]{2}{*}{ Upper back } & $n$ & 201 & 26 & 217 & 10 & 222 & 5 & 225 & 2 \\
\hline & $\%$ & 88.5 & 11.5 & 95.6 & 4.4 & 97.8 & 2.2 & 99.1 & 0.9 \\
\hline
\end{tabular}

Subgroup analysis with Bonferroni correction, $p<0.001$

Since a score of 2 in the chest region contributes to the diagnosis of hirsutism in approximately half of all the patients, its presence is significant and cannot be neglected. However, the inspection of this region in conservative societies is not easy. A smaller-scale study from Turkey yielded similar results to our findings. In a study including 65 patients with hirsutism, Hassa et al. reported a low effect of the upper abdomen, upper arm, and upper back on the total FG score and suggested that these regions could be ignored [8].

Studies have been conducted in different countries to examine women with hirsutism using the $\mathrm{mFG}$ scoring system. Regions of more prominent involvement were determined as the upper lip, chin, lower abdomen, and thighs in Dutch women [9]; lower abdomen, thigh, upper lip, and upper arm in Chinese women [10]; lower abdomen, upper lip, chin, and thighs in Iraqi women [11]; face, chest, and lower abdomen in Pakistani [12] and Indian [13] women; and face, lower abdomen, chest, and thighs in Iranian women [14]. In all these societies and our population, hair growth was concentrated in similar localizations, except for the upper arm in Chinese women.

Based on the fact that approximately $90 \%$ of patients in our study had a terminal hair score of $\geq 1$ in each of the chin, thigh, upper lip or chin, thigh, lower abdomen combinations, the examination of these three regions is useful in estimating that the total mFG score is above 8. In our study, $89 \%$ of the patients were determined to reach a score

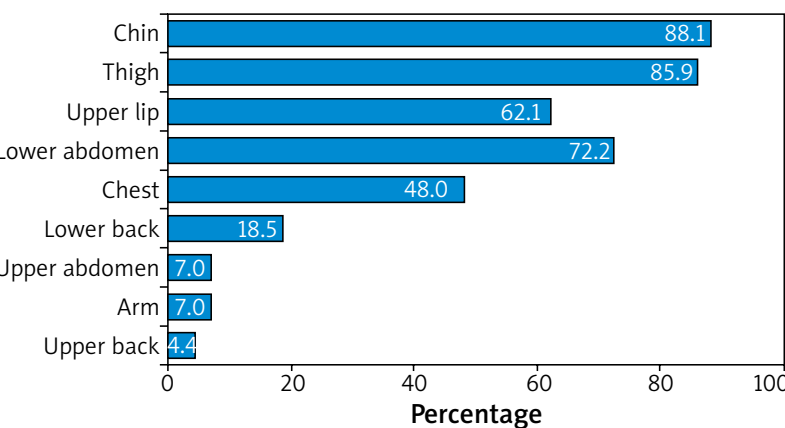

Figure 3. Distribution of patients with a hair growth score of $\geq 2$ according to the body regions

accepted as hirsutism (i.e., 8 and above) with the combination of the chin, thigh, and lip regions, and $87.2 \%$ with the combination of the chin, thigh, and lower abdomen regions. When we examined the average score of hair growth intensity in these regions, it was 2.85 for the chin, 2.86 for the thighs, 1.99 for the lip, and 2.08 for the lower abdomen, confirming this finding. This shows that in our population, examining the chin and thigh regions is sufficient to reach a total mFG score of 6, and when the upper lip or lower abdomen is added to this evaluation, a score of 8 and above can be reached in approximately $90 \%$ of patients. Thus, in addition to the chin and thighs, which are the main regions 
for this evaluation, the lip and lower abdomen regions can contribute equally to the total score.

A few studies have been conducted in different populations with the aim of simplifying the mFG score, as in our study. In a study conducted in Alabama, Knochenhauer et al. found that the examination and scoring of hair growth on the chin or lower abdominal region alone were highly sensitive predictors for the diagnosis of hirsutism. They determined all women with an FG score of $\geq 2$ in any of these body regions and a total score of $\geq 8$ [15]. In an epidemiologic study from China, it was suggested that scores of $\geq 2$ on the lower abdomen, thighs, and upper lip, which are the regions that make the strongest contribution to hirsutism, could be used to predict hirsutism at high sensitivity and specificity [10]. Cook et al., carrying out a two-centre study in Alabama and Los Angeles, determined that the best predictor of the total mFG score was the upper abdomen, lower abdomen, and chin combination or the upper abdomen, lower abdomen, and thigh combination [16]. More recently, in a study from Iran, the authors concluded that evaluating terminal hair growth on the chin or lower abdomen to predict hirsutism seemed to be an acceptable screening method [17]. The body regions we found to be prominent in the diagnosis of hirsutism were similar to those reported by previous studies evaluating different populations, except for the upper abdomen.

Even though $\mathrm{mFG}$ scoring is a subjective hair examination method, it is more difficult to overlook $\geq 2$ hair growth intensity, but grade 1 hair growth may be overlooked according to the region and the evaluation may differ among physicians. Therefore, a score of $\geq 2$ appears to indicate considerable hair growth intensity. In our study, the hair growth intensity score was $\geq 2$ in $75.3 \%$ of the patients when the chin and thighs were evaluated together. The prominence of the same regions also in this evaluation strengthened our findings.

\section{Conclusions}

We propose that four regions including the chin, thighs, upper lip, and lower abdomen stand out in simplifying the mFG scale, and that the remaining four regions including the upper abdomen, lower back, upper back and upper arm can be disregarded in Turkish patients. The thigh and chin regions seem to be the first locations that should be examined to predict hirsutism. We can easily reach this conclusion based on our results from the large-scale study we conducted with the Turkish population.

At a time when we need to follow social distancing rules in daily life, including the hospital environment, a practical approach to the $\mathrm{mFG}$ scoring system will facilitate the work of many physicians, including dermatologists. We consider that this practical approach will shorten the patient's duration of stay in the outpatient clinic and reduce transmission. Thus, dermatologists will be able to provide health services that minimize transmission risk during the ongoing pandemic by also screening hirsutism when evaluating patients with other dermatologic conditions such as acne and androgenetic alopecia.

\section{Acknowledgments}

We would like to thank Dr. Zübeyde Arat, who contributed to obtaining the statistical data and creating the figures for the study.

\section{Conflict of interest}

The authors declare no conflict of interest.

\section{References}

1. Ferriman D, Gallwey JD. Clinical assessment of body hair growth in women. J Clin Endocrinol Metab 1961; 21: 1440-7.

2. McKnight $E$. The prevalence of "hirsutism" in young women. Lancet 1964; 1: 410-3.

3. Escobar-Morreale HF, Carmina E, Dewailly D, et al. Epidemiology, diagnosis and management of hirsutism: a consensus statement by the Androgen Excess and Polycystic Ovary Syndrome Society. Hum Reprod Update 2012; 18: 146-70.

4. Yildiz BO, Bolour S, Woods K, et al. Visually scoring hirsutism. Hum Reprod Update 2010; 16: 51-64.

5. Hatch R, Rosenfield RL, Kim MH, et al. Hirsutism: implications, etiology, and management. Am J Obstet Gynecol 1981; 140: 815-30.

6. Azziz R, Carmina E, Sawaya ME. Idiopathic hirsutism. Endocr Rev 2000; 21: 347-62.

7. Revised 2003 consensus on diagnostic criteria and longterm health risks related to polycystic ovary syndrome (PCOS). Hum Reprod 2004; 19: 41-7.

8. Hassa H, Tanir HM, Yildirim A, et al. The hirsutism scoring system should be population specific. Fertil Steril 2005; 84: 778-80.

9. Derksen J, Moolenaar AJ, Van Seters AP, et al. Semiquantitative assessment of hirsutism in Dutch women. Br J Dermatol 1993; 128: 259-63.

10. Li R, Qiao J, Yang D, et al. Epidemiology of hirsutism among women of reproductive age in the community: a simplified scoring system. Eur J Obstet Gynecol Reprod Biol 2012; 163: 165-9.

11. Hussein RN, Hamdi KIA, Mansour AA. The contribution of new areas to the total hirsutism scores in basrah hirsute women. Diseases 2017; 5: 32.

12. Malik LM, Khursheed K, Haroon TS, et al. An aetiological study of moderate to severe hirsutism. Pak J Med Sci 2007; 23: 167-71.

13. Sharma D, Shanker V, Tegta G, et al. Clinico-investigative profile of patients of hirsutism in a tertiary level institution. Int J Trichology 2012; 4: 69-74.

14. Ansarin H, Aziz-Jalali MH, Rasi A, et al. Clinical presentation and etiologic factors of hirsutism in premenopausal Iranian women. Arch Iran Med 2007; 10: 7-13.

15. Knochenhauer ES, Hines G, Conway-Myers BA, et al. Examination of the chin or lower abdomen only for the prediction of hirsutism. Fertil Steril 2000; 74: 980-3.

16. Cook H, Brennan K, Azziz R. Reanalyzing the modified Ferriman-Gallwey score: is there a simpler method for assessing the extent of hirsutism? Fertil Steril 2011; 96: 1266-70.

17. Rashidi H, Parizi ZT, Mohammadi M. Evaluation of only the chin or lower abdomen for predicting hirsutism. Indian J Endocrinol Metab 2013; 17: 896-8. 